•论坛・生态系统原真性和完整性专题

\title{
中国国家公园原真性与完整性概念及其评价框架
}

\author{
赵智聪(1) 1,2 杨锐(1, $2^{*}$
}

1. 清华大学国家公园研究院, 北京 $100084 ; 2$. 清华大学建筑学院, 北京 100084

摘要: 国家公园保护管理的首要目的是有效保护国家重要自然生态系统原真性和完整性。我们提出了面向管理的中国国家公 园原真性与完整性概念，国家公园原真性是指国家公园内生态系统及构成国家公园价值或与国家公园价值紧密联系的自然 与文化要素保持在原生状态, 强调“不受损”; 国家公园完整性是指国家公园具有足够面积和充分的组成要素, 以维持生态系 统的结构、功能与过程, 维持自然区域的价值与特征, 强调“不缺失”。我们还提出了国家公园原真性和完整性评价框架, 从 自然度、气候变化强度、传统文化及其载体的真实性、人类干扰程度、人与自然和谐关系的原真性 5 个方面评价国家公园原 真性; 从生态系统组成完整性、生态系统结构完整性、生态系统功能完整性、景观组成要素完整性和文化景观的完整性5个 层面评价国家公园完整性。此外, 我们还提出了国家公园原真性与完整性保护的建议, 包括重塑保护管理目标、更新保护管 理理念与提升保护管理技术3个方面。国家公园原真性与完整性的讨论尚未成熟，对评价指标设定、指标间的相互关系、保 护管理技术等的研究均尚待深入。

关键词: 国家公园; 原真性; 完整性; 生态系统; 保护管理

赵智聪, 杨锐 (2021) 中国国家公园原真性与完整性概念及其评价框架. 生物多样性, 29, 1271-1278. doi: 10.17520/biods.2021287.

Zhao ZC, Yang R (2021) The concept of national park authenticity and integrity in China and its evaluation framework. Biodiversity Science, 29, 1271-1278. doi: $10.17520 /$ biods.2021287.

\section{The concept of national park authenticity and integrity in China and its evaluation framework}

Zhicong Zhao ${ }^{(1) 1,2}$, Rui Yang ${ }^{\left(D 1,2^{*}\right.}$

1 Institute for National Parks, Tsinghua University, Beijing 100084

2 School of Architecture, Tsinghua University, Beijing 100084

\section{ABSTRACT}

Aims: The primary purpose of national park conservation and management is to effectively protect the authenticity and integrity of ecosystems, as well as natural and cultural resources of national importance. In this study, we aim to propose the concept of authenticity and integrity for national park management in China and establish a framework for authenticity and integrity evaluation based on the closed loop of value identification, value authenticity, integrity assessment, authenticity and integrity conservation, and the management strategy of monitoring and feedback.

Methods: The primary research method is a review of previous literature on ecosystem integrity, ecosystem authenticity, and conservation management strategies of World Heritage sites, combining them with: practical experience; proposing concepts; systematically analyzing the connotations of the proposed definitions of authenticity and integrity; constructing an evaluation index system; proposing logical relationships among the indicators; and proposing conservation management recommendations based on the concepts and evaluation framework.

Results: In this paper, we propose the authenticity and integrity of China's national parks. National park authenticity means that the ecosystems within a national park and the natural and cultural elements that constitute or are closely associated with national park values are maintained in their original state, with emphasis on "unimpaired". National park integrity refers to the ability of national parks to maintain the structure, functions, and processes of ecosystems as well as the values and characteristics of natural areas with sufficient area and constituent elements, emphasizing "no

收稿日期: 2021-07-19; 接受日期: 2021-10-25

基金项目: 国家自然科学基金项目(51978365)和教育部人文社科项目(21YJCZH240)

* 通讯作者 Author for correspondence. E-mail: yrui@tsinghua.edu.cn 
deficiency”. The significance of the concept of management-oriented national park authenticity and integrity is reflected in three aspects: (1) the distinction between the concepts of authenticity and integrity is helpful for managers to clarify the goals of national park management, (2) using the requirements of authenticity and integrity to comprehensively cover the value system is conducive to the formation of a complete governance and management path of national parks, and (3) specifying the evaluation indexes of the authenticity and integrity of national parks is conducive to judging the effectiveness of national park management more effectively. We propose a framework for evaluating the authenticity and integrity of national parks. To evaluate the authenticity of national parks in five aspects: naturalness, intensity of climate change, authenticity of traditional culture and its carriers, degree of human interference, and authenticity of the harmonious relationship between human beings and nature. The integrity of national parks is evaluated in five dimensions: ecosystem composition integrity, ecosystem structural integrity, ecosystem functional integrity, landscape composition integrity, and cultural landscape integrity. We propose suggestions for the conservation and management of the authenticity and integrity of China's national parks, and those are presented in terms of reshaping objectives, updating concepts and enhancing technologies. The issues of authenticity and integrity of national parks have not yet been discussed in depth, and research on the setting of evaluation indexes, the interrelationship among evaluation indicators, and specific suggestions for conservation and management are yet to be further developed.

Conclusion: This paper proposes a management-oriented concept of authenticity and integrity of Chinese national parks and a framework for authenticity and integrity evaluation. This provides a theoretical basis for establishing a complete management closure loop for Chinese national park management practices. The significance of the concept of authenticity and integrity, the index system of evaluation, and the management suggestions based on these are discussed throughout the paper.

Key words: national park; authenticity; integrity; ecosystem; management

我国国家公园保护管理的首要目标应是保护 国家公园价值的原真性与完整性。中共中央办公 厅、国务院办公厅印发的《建立国家公园体制总体 方案》明确提出国家公园建设的指导思想应“以加 强自然生态系统原真性、完整性保护为基础”, 主要 目标包括“国家重要自然生态系统原真性、完整性 得到有效保护” (http://www.gov.cn/zhengce/2017-09 /26/content_5227713.htm)。2020年12月颁布的国家 标准《国家公园设立规范》(GB/T 39737-2020)规定 了我国国家公园的设立须满足的条件有“国家代表 性、生态重要性、管理可行性”。其中, 生态重要性 包括生态系统完整性、生态系统原真性和面积规模 适宜性共3项指标(http://std.samr.gov.cn/gb/search/gb Detailed?id=B74670820869408EE05397BE0A0A6D 4B)。“健全国家公园保护制度” 是十九届四中全会提 出的国家管理体系和管理能力现代化的重要内容 (http://www.qstheory.cn/dukan/qs/2020-01/01/c_1125 402833.htm)。如何将国家公园所代表的我国国土上 生态系统最重要、自然景观最精华、生物多样性最 富集的部分原真、完整地保护好, 是体现国家治理 能力的重要方面。

我国国家公园的定义为“由国家批准设立并主 导管理, 边界清晰, 以保护具有国家代表性的大面 积自然生态系统为主要目的, 实现自然资源科学保
护和合理利用的特定陆地或海洋区域” (http://www.gov.cn/zhengce/2017-09/26/content_5227 713.htm)。从定义来看, “大面积自然生态系统” 是 国家公园保护的主要对象, 其他自然资源的科学保 护也应同时考虑。从生态学领域考察, 生态系统原 真性、生态系统完整性的概念都有了较为广泛和深 入的讨论(陈昌笃和李迪华, 2003), 尤其是对生态系 统完整性的内涵、评价等领域的研究, 近年得到关 注, 又因为遥感、地理信息系统、生态学模型等技 术的进步而发展出一系列的评价模型和指标。

然而, 对于管理者而言, 国家公园管理所要面 对的是一个复杂的系统, 不仅包括自然生态系统本 身, 还包括承载自然生态系统的地质背景、地理环 境及其所产生的景观或审美认知, 乃至人类社会的 遗迹与现今社会文化背景下的公众对价值的认知 等(Poulios, 2010; 彭琳和杨锐, 2018)。仅以生态系 统的原真性与完整性来要求或衡量国家公园的保 护管理, 显得捉襟见肘。

我国国家公园的设立、保护与管理实践迫切需 要建立以价值保护为目标的逻辑起点, 形成对国家 公园价值的完整性与真实性状态为保护目标, 以维 持、加强国家公园价值的完整性与真实性为主要手 段, 以完整性、原真性保护成效为监测反馈指标的 管理体系。国家公园原真性与完整性的概念内涵、 
评价标准的研究, 成为亟待建立的“价值识别-原真 性与完整性评价一原真性与完整性的保护恢复-原 真性与完整性保护恢复效果反馈”管理闭环的基本 前提。

本文提出面向管理的国家公园原真性与完整 性概念, 在生态系统完整性和原真性概念基础上, 将国家公园的整体空间范围作为研究对象, 区分原 真性与完整性, 并针对原真性与完整性各自关注的 不同重点, 提出评价框架与评价指标, 从而为提升 国家公园原真性与完整性提出管理建议。

\section{完整性与原真性相关概念内涵}

\section{1 完整性及相关概念}

与完整性相关的概念在中文环境中相继出现 过生物完整性、生态完整性、生态系统完整性等用 词。多数学者将英文“ecological integrity”翻译为生 态系统完整性, 并已有诸多学者对其概念起源、发 展、定性定量评价方法及其在保护地领域的应用等 研究进展进行了综述。

一般认为, Leopold (1949年)在其关于土地伦理 学的文章中首先提出了生态系统完整性的相关概 念, 即“人类活动朝着保护生物群落完整性、稳定性 和美感等方向发展时是正确的, 相反则是错误的”。 这一概念基于 “土地伦理” 定义, 却并没有在生态学 意义上做出进一步的阐述。之后Karr和Dudley (1981) 明确给出生态系统完整性的定义, 即“完整性是支 持和保持一个平衡的、综合的、适宜的生物系统的 能力”。

生态系统完整性被认为是正常演化的生态系 统本身应该具备的一种属性, 这一属性有助于生态 系统本身维持其应有的能力。生态系统完整性“反 映生态系统在外来干扰下维持自然状态、稳定性和 自组织能力的程度”, 并逐渐与生态系统的稳定性、 生态系统的自组织能力、生态系统健康等概念联系 起来。IUCN提出, 生态完整性是“维持生态系统的 多样性和质量, 加强它们适应于变化并供给未来需 求的能力”(https://www.iucn.org/sites/dev/files/iucn-g lossary-of-definitions_en_2021.05.pdf)。

在我国《国家公园设立标准》中, 生态系统完 整性被阐述为“自然生态系统的组成要素和生态过 程完整, 能够使生态功能得以正常发挥, 生物群 落、基因资源及未受影响的自然过程在自然状态下
长久维持。应符合的特征包括生态系统健康、生态 功能稳定、生物多样性丰富、具有顶级食肉动物存 在的完整食物链或迁徙洄游动物的重要通道、越冬 (夏)地或繁殖地”, 这一定义反映了目前对于生态系 统完整性较为普遍的认识。

这一概念在自然保护地领域最为典型的应用 是加拿大国家公园系统提出的国家公园“生态完整 性评估” (Parks Canada Agency, 2005): 不仅明确界 定了“生态完整性”的定义, 也提出了适用于加拿大 国家公园的完整性评价框架、指标和方法。世界遗 产委员会提出的“完整性”概念相对宽泛, 是针对世 界遗产地这一空间范围如何管理提出的。《实施世 界遗产公约的操作指南》 (The Operational Guidelines for the Implementation of the World Heritage Convention, 以下简称世界遗产《操作指 南》)阐明了世界自然遗产应满足的4条标准, 也应 分别符合完整性评价的管理要求 (http://whc.unesco.org/en/guidelines/), 可以概括为: (1)面积足够大, 以包括全部或大多数相关要素、特 征、过程和功能等; (2)具备充分的保护管理规划或 相关措施，以保证世界遗产的完整性受到适当的、 充分的保护和管理(张成渝, 2004)。这一概念提出了 针对世界遗产地的申报、保护与管理而言相对容易 操作的路径, 即阐述受保护的面积是否足够大, 以 包括所有应该被保护的要素及其相互关系。相比较 于生态系统完整性的概念, 世界遗产的完整性补充 了在地质地貌、美学价值等层面对是否“未缺失” (intactness) 的考虑, 可为国家公园完整性概念提供 借鉴。

\section{2 原真性相关概念}

与完整性相比, 对于生态系统原真性的讨论远 没有那么深入。在恢复生态学领域, 出现了生态系 统原真性(ecological authenticity)的概念, 并分为自 然原真性 (natural authenticity) 与历史原真性 (historical authenticity)(何思源和苏杨, 2019)。这一 概念是在恢复生态学要设法使生态系统回到原来 的正常轨迹的语境下出现的, 因此, “原来的” 状态 成为生态恢复设计的理想出发点, 也变得非常关 键。“自然原真性” 是指生态系统回到健康状态, 但 是不考虑生态系统是否精确地反映出它的历史结 构和组成; “历史原真性” 是指生态恢复需要让恢复 后的生态系统与一个历史参考状态相匹配(Clewell, 
2000)。从恢复生态学的视角考察, 生态系统的原真 性是生态恢复过程的某一个端点(endpoint), 生态系 统的自身发展会存在多个端点, 或多种可能性, 恢 复的过程是促使其沿着某一轨迹开始演化。世界上 大部分生态系统目前正处在退化之中, 联合国《生 物多样性公约》(Convention on Biological Diversity) 认为, 生态恢复是管理或帮助一个退化、受损或破 坏的生态系统恢复的过程, 以维持生态系统恢复力 和保护生物多样性(https://www.cbd.int/doc/decisions /cop-13/cop-13-dec-05-en. pdf) (CBD, 2016)。因此生 态恢复工作更关注如何让具体的场地、景观或生态 系统回到某种较为平衡的、健康的状态, 生态系统 的原真性成为生态恢复背后暗含的目标状态, 并没 有发展出明确的概念体系与评价方法。

在世界遗产领域, “authenticity”一词用于评价 文化遗产的真实性。在世界遗产《操作指南》中认 为, 想要保护世界遗产的真实性, 应从认识和理解 遗产价值开始, 不仅包括遗产本身的特征、形成历 史等, 还包括这些信息的意义和来源的真实性。在 被称为《奈良真实性文件》(Nara Document)的文化 遗产保护的重要共识中, 对文化遗产的真实性评价 从“形式与设计、材料与实质、利用与作用、传统 与技术、位置与环境、精神与感受”等6 个方面展开 (WHC, 1994), 这些方面对文化遗产的针对性极强, 因此对自然遗产而言真实性概念并没有广泛应用。

美国国家公园管理中有一个与原真性相关的 概念, 即“不受损害的” (unimpaired), 在美国国家公 园管理局《组织法》(NPS Organic Act)中明确指出, 美国国家公园管理局的使命是让自然与文化资源 为后代“不受损害”地保留下来(https://www.justice.g ov/enrd/nps-organic-act。但是, 相关研究对这个概念 多是以历史视角来讨论管理中的政策倾向, 例如, 引入多少访客活动能保证国家公园不受损害, 大型 基础设施建设是否妨碍了这种 “不受损害”的状态 等。在美国国家公园系统中, 这一概念的应用更多 倾向于辅助国家公园的园长(superintendents)进行 价值判断和决策(Keiter, 2013)。对于这一概念的讨 论实际上并没有与原真性或完整性直接对应起来, 但其对美国国家公园保护管理目标状态的指导意义 是明显的, 且“不受损害”的对象是整个国家公园。

在我国《国家公园设立规范 $(G B / T$ 39737-2021)》中, 生态系统原真性是指生态系统与
生态过程大部分保持自然特征和自然演替状态, 自 然力在生态系统和生态过程中居于支配地位。主要 通过规定自然区域的面积占比、人类生产生活区域 的面积占比、人类居住区域的面积占比等指标来描 述。在关于该国标的研究中, 也讨论过用“荒野度” 来考察原真性, 即“拥有大面积高质量荒野, 生态 系统和生态过程处于高质量的自然状态”, 但并未 在该国标中全部采用。

\section{3 完整性与原真性的关系}

目前尚未有文献将生态系统完整性和生态系 统原真性两个概念并置并系统阐述。关于青藏高原 国家公园群的研究认为完整性与原真性是一个整 体的概念, 并构建了评价二者的指标体系(刘晓娜 等, 2021)。也有研究认为, 原真性和完整性均涉及 时空尺度和自然生态系统自组织功能, 二者的保护 是国家公园的首要功能(何思源和苏杨, 2019)。在国 家公园设立标准的前期研究中, 原真性被阐述为荒 野度和自然度两个层次, 荒野度强调某一区域未受 人为控制的程度, 自然度指物种分布与组成、演替 状态是自然力量主导的; 完整性指要素完整和面积 足够大, 包括物理环境要素、生境要素和生态过程 要素; 面积足够大则表现为荒野面积与自然生境面 积均足够大。可见完整性和原真性概念也没有明确 区分开。

综上, 无论从上述概念层面的考察还是评价框 架方面的研究都反映出对于原真性与完整性概念 认识的如下特征：(1)随着完整性概念的不断发展, 其包含的内容逐渐丰富, 从关注生态系统的组成要 素是否完整, 到生态系统的结构与功能, 也考虑生 态系统所具有的自组织能力、韧性特征、健康状态 等内容, 同时也开始关注和原始自然相比或与某一 参照的生态系统相比是否完整, 与生态恢复领域关 注的生态系统原真性概念逐渐接近; (2)生态系统原 真性概念并未形成相对系统的概念内涵、评价框架, 尚无合适的指标体系予以评价; (3)在国家公园、自 然保护地、世界自然遗产等领域, 只讨论生态系统 原真性与完整性尚不足以支撑其管理行为。

\section{中国国家公园原真性与完整性概念}

\section{1 内涵}

本文提出了中国国家公园原真性和国家公园 完整性概念。 
国家公园原真性是指国家公园内生态系统及 构成国家公园价值或与国家公园价值紧密联系的 自然与文化要素保持在原生状态。原真性概念强调 “不受损”, 对于自然生态系统而言, 人类干扰程度 受到严格控制, 自然干扰维持其原本状态, 生态系 统本身具有自组织能力。与自然生态系统相关的非 生物要素和文化要素对自然生态系统维持其原本 的能力具有重要作用, 因此也成为原真性保护的对 象。非生物要素通常包括地质地貌、光、温、水、 土壤等, 文化要素包括人们对自然的认识、与自然 相关的行为, 以及与自然相互作用产生的结果, 如 自然圣境(sacred natural sites)中的自然崇拜观念、朝 圣或禁忌行为, 由此产生的建构筑物或有明确传统 边界的自然地域; 传统生计中对于自然资源的可持 续利用行为等。

国家公园完整性是指国家公园具有足够面积 和充分的组成要素, 以维持生态系统的结构、功能 与过程, 维持自然区域的价值与特征。完整性概念 强调“不缺失”, 对于生态系统强调其组成、结构与 功能的完整, 对于非生物要素强调体现景观价值的 内容被完整包括, 对于与自然紧密联系的文化要素 则强调载体的完整与不被割裂。

\section{2 意义}

在国家公园“价值识别－原真性与完整性评价原真性与完整性的保护恢复-原真性与完整性保护 恢复效果反馈” 的管理框架下, 价值识别为国家公 园管理设立了逻辑起点, 价值的原真性与完整性评 价为管理政策构建了基础, 同时也为管理成效的评 价提供了依据。提出面向管理的国家公园原真性与 完整性概念的意义体现在以下 3 个方面:

(1)将原真性与完整性概念区分出来, 有利于 管理者明确国家公园管理的目标。尽管在生态系统 管理方面, 完整性与原真性的区别仍有待进一步探 讨, 但对于管理者而言, 用完整性强调国家公园应 受到全面的、充分的保护; 用原真性强调人类干扰 程度低, 国家公园内必须是自然或近自然的状态, 在全面、充分的基础上, 国家公园受保护的目的是 维持自然生态系统原本的状态。将原真性与完整性 分开讨论, 并不是要抛弃基于生态学基本原理的生 态系统完整性和生态系统原真性的科学内涵, 而是 借用这些理论, 进一步扩展完整性和原真性保护的 对象。
(2)用原真性与完整性要求来全面覆盖价值体 系, 有利于形成完整的管理路径。经过识别并确认 的国家公园的价值, 都应满足原真性与完整性要 求。国家公园价值不仅包括生态系统, 还应包括国 家公园中包含的地质资源价值, 以及与生态系统密 切相关的原住民的传统文化、传统生计等文化资源 价值, 人类社会长期积淀的对于自然的审美认识 等。从而形成“价值识别-价值的原真性与完整性评 估-保护管理策略-保护管理成效反馈”的国家公园 管理技术路径。

(3)明确国家公园原真性与完整性的评价指标, 有利于更好地判断国家公园管理效果。国家公园原 真性与完整性的概念明确后, 则应以定义为出发点, 研制可以评估原真性与完整性的评价框架与指标, 从而在纵向上对一段时间后的国家公园管理效果 进行评估, 即国家公园的原真性和完整性是否得以 维持、是否得以提升; 横向上对同一生物地理区内 的国家公园乃至各种类型的自然保护地进行评估 和比较, 从而判断保护力量的投入应如何倾斜。

\section{3 国家公园原真性与完整性评价框架}

\section{1 评价体系与指标的构建原则}

国家公园原真性与完整性的评价体系与指标 尚处于研发阶段, 尚未形成明确的、达成共识的评 价体系。因此, 需要首先从科学内涵与面向管理两 个层面明确评价体系构建的基本原则。

在科学内涵层面, 应从完整性与原真性的概念 出发构建评价体系。国家公园原真性与完整性概念 一方面强调其对生态系统而言应面向生态系统的 结构与功能两个维度, 这是国家公园管理的基础和 重点; 另一方面, 国家公园是一个复杂的地域系统, 尤其是在中国传统文化和生态文明的背景下, 基于 生物多样性、文化多样性、社会-生态系统等多种理 论体系, 也应强调对国家公园价值的评价应将国家 公园视为一个整体、一个系统而进行全面评价。因 此, 应充分继承生态学相关领域、世界遗产相关领 域等对于原真性和完整性评价的相关研究, 尽可能 选取具有生态模型基础、定量关系较为明确的评价 指标; 同时, 也应注重新的评价指标的研发, 以充 分反映我国国家公园特征。

在面向管理层面, 国家公园原真性与完整性的 评价应注重可用性。在我国, 国家公园管理仍属新 
事物，尽管有诸多国际经验教训可资借鉴，但我国 国家公园管理仍然面对时代背景、地理环境、动力 机制、基本目标、地位规模和管理难度等方面的特 殊性，因此，服务于我国国家公园管理的完整性与 原真性评价，应注重指标的快速可评、横向同一区 域内与纵向不同时段的可比性、对保护管理具有指 导意义等面向应用的管理需求。

\section{2 国家公园原真性与完整性评价框架}

已有研究表明, 生态系统完整性评价指标与方 法已从水生生态系统完整性评价的生物完整性指 数(Index of Biological Integrity, IBI) (Karr \& Dudley, 1981), 扩展到陆地生态系统的评价方法(Kay, 2001) 等。我国学者也提出了生物多样性、连通性和破碎
度、生态系统产品/服务、人类活动干扰等指标体系 (黄宝荣等, 2006; 燕乃玲和虞孝感, 2007)。

本研究构建了由一级指标和二级指标构成的 国家公园原真性和完整性评价指标体系。一级指标 决定了评价的基本维度, 二级指标仅进行了举例说 明, 完整的指标体系尚待进一步研究, 详见表 1 。

国家公园原真性评价包括5个一级指标。自然 度指标、气候变化强度和传统文化及其载体的真实 性指标强调国家公园的“状态”。自然度指标强调自 然要素的自然状态, 传统文化及其载体真实性指标 强调文化要素的真实状态, 气候变化强度强调在较 长时间周期内，国家公园的某些典型特征是否发生 了不符合长期演化规律的“突变”或“巨变”，这一指

表1 国家公园原真性与完整性评价框架

Table 1 Framework for evaluating the authenticity and integrity of China's national parks

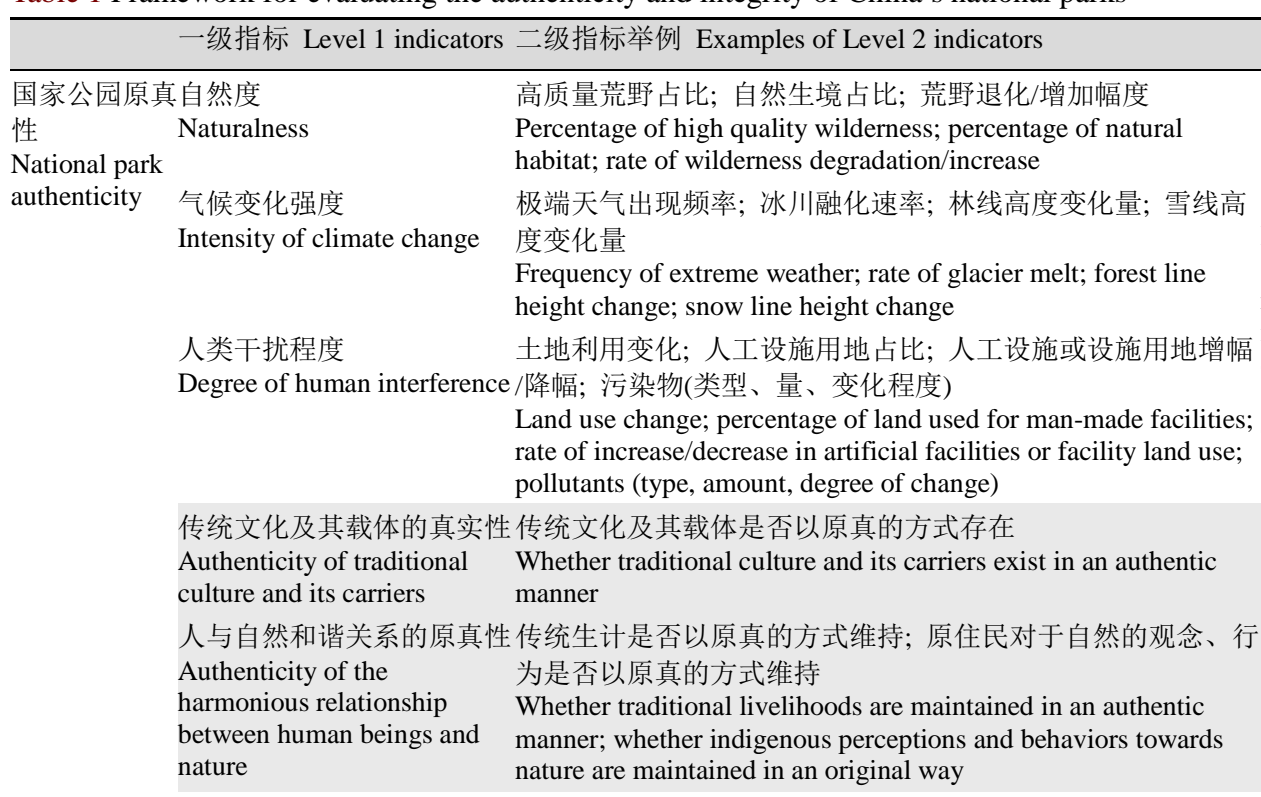

国家公园完整生态系统组成完整性 性 Ecosystem composition National park integrity

integrity 生态系统结构完整性

Ecosystem structural integrity Nutrient structure; habitat quality

生态系统功能完整性 Ecosystem functional integrity

景观组成要素完整性 Landscape composition integrity

文化景观的完整性 Cultural landscape integrity service functions

否被有效保护 effectively protected
方法 Methods

基于遥感影像的判别、监测、制图、 运算、模拟; 基于田野调查的识别、 监测、实测、判定; 基于历史文献

与数据的分析

Identification, monitoring, mapping, arithmetic, and simulation based on remote sensing images; identification, monitoring, actual measurement, and determination based on field surveys; analysis based on historical documents and data.

观察、访谈、田野调查

Observations, interviews, fieldwork

行

本地重要物种是否被包括; 物种丰富度; 种群数量; 种群动态 基于遥感影像的判别、监测、制图、 Whether locally important species are included; species richness; 运算、模拟; 基于田野调查的识别、 population size; population dynamics

监测、判定

Identification, monitoring, mapping, arithmetic, and simulation based on remote sensing images; identification, monitoring, actual measurement, and determination based on field surveys

Succession; trophic levels; decomposition capacity; ecosystem

地质地貌价值载体是否被完整包括; 构成价值的河流的源头是

Whether geomorphic resources are included in their entirety; whether the source of the river that constitutes the value is

传统保护边界是否完整纳入; 原住民传统组织结构是否被分割观察、访谈、田野调查

Whether traditional conservation boundaries are included intact; Observations, interviews, fieldwork whether the traditional organizational structure of the indigenous people is divided 
标更强调对气候变化敏感的生态系统的关注。人类 干扰程度指标强调国家公园的压力或威胁; 人与自 然和谐关系的原真性指标强调中国国家公园的特 征。总体上, 原真性评价指标注重反映历时性变化, 如果变化的强度、频率在较长时间内是基本稳定的, 则可以认为其原真性较高。

国家公园完整性指标包括5个一级指标。生态 系统组成完整性、生态系统结构完整性和生态系统 功能完整性等3个指标反映了国家公园的核心保护 对象自然生态系统的特征; 景观组成要素完整性指 标强调部分未被自然生态系统包括的非生物要素 的价值是否完整, 如作为审美对象的地质地貌景观, 作为湖泊、瀑布等具有观赏性价值的景观要素的上 游区域是否得到完整保护等; 文化景观的完整性强 调与自然发生 “强联系”的文化要素是否得到完整保 护(赵智聪等, 2013), 如与原住民信仰相关的神山圣 湖所形成的具有行为规定特征的传统保护边界(赵 智聪和王沛, 2021), 是否完整纳入了保护体系等。 完整性指标更注重反映国家公园在空间尺度上是 否得到了完整保护, 构成国家公园价值的要素、价 值的载体、价值的支撑系统可以得到有效保护, 则 可以认为其完整性较高。

\section{4 结论与讨论}

提出面向管理的国家公园原真性与完整性概 念, 目的在于在继承并拓展生态学等领域完整性与 原真性相关概念科学内涵的基础上, 为我国国家公 园管理提供基础依据与逻辑起点。

基于对国家公园原真性和完整性的概念建构, 对我国国家公园管理提出重塑目标、更新理念、提 升技术等层面的建议。(1)重塑保护管理目标。国家 公园管理的目标是保护国家公园的原真性与完整 性, 即自然生态系统不受损、不缺失、能够维持其 自身健康、发挥各种功能的状态。这一目标不仅包 括自然生态系统, 还包括人与自然的长期和谐共 荣。将原真性与完整性作为目标, 意味着对国家公 园保护管理提出了更高要求, 围绕这一目标亟需开 展一系列科学研究与管理体系建设。(2)更新保护管 理理念。国家公园原真性概念强调的是“自然力量”, 国家公园作为应对气候变化与生物多样性丧失重 大危机、保护和传承人与自然和谐相处的典范模式, 如何在管理过程中解锁更多自然潜力、发挥更大自
然力量应成为国家公园管理领域的核心理念。这不 仅是对国际社会 “基于自然的解决方案” (Nature-based Solution, NbS, https://www.iucn.org/th eme/nature-based-solutions)的积极响应, 也是对当 前我国国家公园保护与管理国情的有力回应。面对 国家公园这一复杂系统, 强调自然力量的理念, 指 导管理者采用“再野化”等更为自然的方法来进行脆 弱生态系统的恢复; 引导管理者面对访客需求、经 济发展等压力时, 考虑自然本身健康状态的承载力; 指引公众更好地认识国家公园是大面积的自然生 态系统的本质属性, 从而规避不恰当行为的发生。 (3)提升保护管理技术。应基于国家公园原真性与完 整性保护目标, 构建闭环的保护管理路径。国家公 园的原真性与完整性应是一对具有包容性的概念, 提供充分的探索空间, 与之相关的连通性、㓞性、 生态系统健康等内容应该在原真性与完整性的框 架下得到充分研究、监测, 乃至实验。再野化、大 尺度生态廊道识别与构建、应对气候变化的生物多 样性保护等都是原真、完整的保护国家公园的新技 术与硬技术, 需要持续探索。对于人与自然和谐的 原真性指标、基于传统文化力量来保护自然区域的 文化景观相关内容, 如何调查、评价和监测, 仍需 探索可行路径。

我国国家公园的管理实践已经对原真性、完整 性等议题提出了迫切需求。本研究提出的国家公园 完整性与原真性的概念及其评价框架仅为初步构 想。在界定原真性与完整性的科学内涵方面, 仍然 需要多学科共同努力, 从不同学科视角论证与研发 原真性与完整性的认识方法、监测技术、评价标准; 在原真性与完整性的保护管理方面, 需要利益相关 者的多方参与, 尤其是拥有与自然紧密联系的传统 文化的原住民，应成为原真性保护的重要管理主体。 综合考虑我国国家公园的管理进程与需求, 在原真 性与完整性方面的近期研究重点应包括以下内容: (1)如何进一步明确界定我国国家公园原真性与完 整性科学内涵, 以充分适应我国国家公园现实需求 的同时, 为国家公园保护管理设定理想目标; (2)研 发适合我国国家公园的原真性与完整性评价框架与 指标体系, 既科学准确, 又快速可行, 是这一评价框 架与指标体系研制的重难点问题; (3)针对国家公园 原真性与完整性的保护, 研究制定保护管理策略, 以促进我国国家公园真正成为生态文明建设的典范。 


\section{ORCID}

赵智聪 (D) https://orcid.org/0000-0001-6215-8119

杨锐 (D) https://orcid.org/0000-0001-6212-8753

\section{参考文献}

Chen CD, Li DH (2003) On the biodiversity and the ecological integrity of Wulingyuan district, Hu'nan Province. Acta Ecologica Sinica, 23, 2414-2423. (in Chinese with English abstract) [陈昌笃, 李迪华 (2003) 湖南省武陵源地区的 生物多样性和生态完整性. 生态学报, 23, 2414-2423.]

Clewell AF (2000) Restoring for natural authenticity. Ecological Restoration, 18, 216-217.

Frank JJ (2014) To conserve unimpaired: The evolution of the National Park idea. Environmental History, 19, 606-608.

He SY, Su Y (2019) Authenticity, integrity, connectivity and coordination-conceptual analysis and practical significance for the establishment of the national park system. Environmental Protection, 47(Z1), 28-34. (in Chinese) [何 思源, 苏杨 (2019) 原真性、完整性、连通性、协调性概 念在中国国家公园建设中的体现. 环境保护, 47(Z1), , 28-34.]

Huang BR, Ouyang ZY, Zheng H, Wang XK, Miao H (2006) Connotation of ecological integrity and its assessment methods: A review. Chinese Journal of Applied Ecology, 17, 2196-2202. (in Chinese with English abstract) [黄宝荣, 欧阳志云, 郑华, 王效科, 苗鸿 (2006) 生态系统完整性 内涵及评价方法研究综述. 应用生态学报, 17 , 2196-2202.]

Karr JR, Dudley DR (1981) Ecological perspective on water quality goals. Environmental Management, 5, 55-68.

Kay JJ (2001) The Ecosystem Approach to Monitoring Integrity [M/OL]. Faculty of Environmental Studies, University of Waterloo. http://www.fes.uwaterloo.ca/u/jjkay /HNA/chapter2.html. (accessed on 2021-05-10)

Keiter R (2013) To Conserve Unimpaired: The Evolution of the National Park Idea. Island Press, Washington, DC.

Leopold A (1949) A Sand County Almanac and Sketches Here and There. Oxford University Press, New York.

Liu XN, Liu CL, Zhang CL, Wei Y, Huang BR (2021) Ecosystem integrity and authenticity assessment framework in the Qinghai-Tibet Plateau National Park Cluster. Acta Ecologica Sinica, 41, 833-846. (in Chinese with English abstract) [刘晓娜, 刘春兰, 张从林, 魏钰, 黄宝荣 (2021) 青藏高原国家公园群生态系统完整性与原真性评估框架. 生态学报, 41, 833-846.]

Parks Canada Agency (2005) Monitoring and Reporting
Ecological Integrity in Canada's National Parks, Guiding Principles, Vol. 1. Parks Canada Agency, Quebec, Canada.

Peng L, Yang R (2018) On the holistic value of scenic areas and its identification. Chinese Landscape Architecture, 34(7), 42-47. (in Chinese with English abstract) [彭琳, 杨 锐 (2018) 论风景名胜区整体价值及其识别. 中国园林, 34(7), 42-47.]

Poulios I (2010) Moving beyond a values-based approach to heritage conservation. Conservation and Management of Archaeological Sites, 12, 170-185.

WHC (1994) Nara Conference on Authenticity in Relation to the World Heritage Convention. Tokyo, UNESCO-World Heritage Center, Agency for Cultural Affairs, CCROM, ICOMOS.

Yan NL, Yu XG (2007) Summary comments on ecosystem integrity. Progress in Geography, 26, 17-25. (in Chinese with English abstract) [燕乃玲, 虞孝感 (2007) 生态系统 完整性研究进展. 地理科学进展, 26, 17-25.]

Yang R (2018) A study of the criteria for the establishment of national parks in China. Forestry Construction, (5), 103-112. (in Chinese) [杨锐 (2018) 中国国家公园设立标 准研究. 林业建设, (5), 103-112.]

Zhang CY (2004) Interpretation and analysis for 2 important conceptions of the world heritage convention: Study on the world heritage's authenticity and integrity. Acta Scicentiarum Naturalum Universitis Pekinesis, 40(1), 129-138. (in Chinese with English abstract) [张成渝 (2004) 《世界遗产公约》中两个重要概念的解析与引申一一论世 界遗产的“真实性”和“完整性”. 北京大学学报(自然科学 版), 40(1), 129-138.]

Zhao ZC, Liu XH, Yang R (2013) Identification of the perception and conservation of Chinese national Parks as cultural landscapes. Chinese Landscape Architecture, 29(11), 30-33. (in Chinese with English abstract) [赵智聪, 刘雪华, 杨锐 (2013) 作为文化景观的风景名胜区认知 与保护问题识别. 中国园林, 29(11), 30-33.]

Zhao ZC, Wang P (2021) Research on Cognition Tradition and Spatial Pattern of Sacred Natural Sites of Three-River-Source National Park: Taking Angsai Township of Lancang River Source Park as an Example. Landscape Architecture, 28(4), 117-123. (in Chinese with English abstract) [赵智聪, 王沛, 三江源国家公园自然圣 境认知传统与空间格局研究——以澜沧江源园区昂赛乡 为例. 风景园林, 28(4), 117-123.]

(责任编委: 徐卫华 责任编辑: 周玉荣) 